\title{
Low-lying Quasiparticle Excitations around a Vortex Core in Quantum Limit
}

\author{
N. Hayashi, T. Isoshima, M. Ichioka, and K. Machida \\ Department of Physics, Okayama University, Okayama 700, Japan
}

(Submitted 26 September 1997)

\begin{abstract}
Focusing on a quantum-limit behavior, we study a single vortex in a clean $s$-wave type-II superconductor by selfconsistently solving the Bogoliubov-de Gennes equation. The discrete energy levels of the vortex bound states in the quantum limit is discussed. The vortex core radius shrinks monotonically up to an atomic-scale length on lowering the temperature $T$, and the shrinkage stops to saturate at a lower $T$. The pair potential, supercurrent, and local density of states around the vortex exhibit Friedel-like oscillations. The local density of states has particle-hole asymmetry induced by the vortex. These are potentially observed directly by STM.

PACS number(s): 74.60.Ec, 61.16.Ch, 74.72.-h
\end{abstract}

Growing interest has been focused on vortices both in conventional and unconventional superconductors from fundamental and applied physics points of view. This is particularly true for high- $T_{c}$ cuprates, since it is essential that one understands fundamental physical properties of the vortices in the compounds to better control various superconducting characteristics of some technological importance. Owing to the experimental developments, it is not difficult to reach low temperatures of interest where distinctive quantum effects associated with the discretized energy levels of the vortex bound states are expected to emerge. The quantum limit is realized at the temperature where the thermal smearing is narrower than the discrete bound state levels [1]: $T / T_{c} \leq 1 /\left(k_{\mathrm{F}} \xi_{0}\right)$ with $\xi_{0}=v_{F} / \Delta_{0}$ the coherence length $\left(\Delta_{0}\right.$ the gap at $\left.T=0\right)$ and $k_{\mathrm{F}}\left(v_{F}\right)$ the Fermi wave number (velocity). For example, in a typical layered type-II superconductor $\mathrm{NbSe}_{2}$ with $T_{c}=7.2 \mathrm{~K}$ and $k_{\mathrm{F}} \xi_{0} \sim 70$, the quantum limit is reached below $T<50 \mathrm{mK}$. As for the high- $T_{c}$ cuprates, the corresponding temperature is rather high: $T<10 \mathrm{~K}$ for $\mathrm{YBa}_{2} \mathrm{Cu}_{3} \mathrm{O}_{7-\delta}(\mathrm{YBCO})$.

Important microscopic works to theoretically investigate the quasiparticle spectral structure around a vortex in a clean limit are put forth by Caroli et al. 2], Kramer and Pesch [1], and Gygi and Schlüter [3]. The low-lying excitations are essential to correctly describe low- $T$ thermodynamic and transport properties in the vortex state (or the mixed state). These include anomalous electric or thermal Hall conductivity [4] and mysterious observations of the quantum magnetic $\mathrm{dHvA}$ oscillations [5]; various topics are debated intensively [6]. Yet there has been no serious attempt or quantitative calculation to explore deep into the quantum regime.

The purposes of the present paper are to reveal the quantum-limit aspects of the single vortex in $s$-wave superconductors and to discuss a possibility for the observation of them.

The present study is motivated by the following recent experimental and theoretical situations: (1) The socalled Kramer and Pesch (KP) effect [1],3,7,8,8]; a shrinkage of the core radius upon lowering $T$ (to be exact, an anomalous increase in the slope of the pair potential at the vortex center at low $T$ ) is now supported by some experiments [9]. The $T$ dependence of the core size is studied by $\mu \mathrm{SR}$ on $\mathrm{NbSe}_{2}$ and $\mathrm{YBCO}$ [10], which is discussed later. The KP effect, if confirmed, forces us radically alter the traditional picture [11] for the vortex line such as a rigid normal cylindrical rod with the radius $\xi_{0}$. (2) The scanning tunneling microscopy (STM) experiment on YBCO by Maggio-Aprile et al. [12], which enables us to directly see the spatial structure of the low-lying quasiparticle excitations around the vortex, arouses much interest. They claim that surprisingly enough, there exist only a few discretized bound-state levels in the vortex core, i.e., the vortex is almost "empty." It resembles our naive image for conventional $s$-wave superconductors where in the quantum limit a few quantized levels of the bound states remain inside the bulk energy gap $\Delta_{0}$. (3) The theoretical situation on this subject 12 is still very confusing; Some 13] claim that the bound-state energy levels are not discretized for $d$-wave pair, but discretized for $s$-wave pair. Some [14] claim the discretizedlike structure even for the former. For $s$-wave case, where the formulation of the problem is well defined, we should establish our understanding of the vortex structure in the quantum limit. (4) Lastly, we are motivated by a curiosity; Previously we have calculated the local density of states (LDOS) for $s$-wave pair on the basis of the quasiclassical (Eilenberger) theory [15], successfully applied to the STM observations on $\mathrm{NbSe}_{2}$ done by Hess et al. [16]. We are particularly interested in what happens in LDOS at further lower $T$, say, below $50 \mathrm{mK}$ deep into the quantum limit, at which it may be now feasible to perform STM experiments.

Prompted by these motivations, we self-consistently solve the Bogoliubov-de Gennes (BdG) equation, which is one of the most fundamental microscopic equations of superconductivity and contains fully quantum effects. We start with the BdG equations for the quasiparticle wave functions $u_{j}(\mathbf{r})$ and $v_{j}(\mathbf{r})$ labeled by the quantum number $j$ : 


$$
\begin{array}{r}
{\left[\frac{-1}{2 k_{\mathrm{F}} \xi_{0}} \nabla^{2}-E_{\mathrm{F}}\right] u_{j}(\mathbf{r})+\Delta(\mathbf{r}) v_{j}(\mathbf{r})=E_{j} u_{j}(\mathbf{r}),} \\
-\left[\frac{-1}{2 k_{\mathrm{F}} \xi_{0}} \nabla^{2}-E_{\mathrm{F}}\right] v_{j}(\mathbf{r})+\Delta^{*}(\mathbf{r}) u_{j}(\mathbf{r})=E_{j} v_{j}(\mathbf{r}),
\end{array}
$$

in a dimensionless form, where $\Delta(\mathbf{r})$ is the pair potential and $E_{\mathrm{F}}\left(=k_{\mathrm{F}} \xi_{0} / 2\right)$ the Fermi energy. The length (energy) scale is measured by $\xi_{0}\left(\Delta_{0}\right)$. For an isolated single vortex in an extreme type-II superconductor, we may neglect the vector potential in Eq. (1). The pair potential is determined self-consistently by

$$
\Delta(\mathbf{r})=g \sum_{\left|E_{j}\right| \leq \omega_{\mathrm{D}}} u_{j}(\mathbf{r}) v_{j}^{*}(\mathbf{r})\left\{1-2 f\left(E_{j}\right)\right\}
$$

with the Fermi function $f(E)$. Here, $g$ is the coupling constant and $\omega_{\mathrm{D}}$ the energy cutoff, which are related by a BCS relation via the transition temperature $T_{c}$ and the gap $\Delta_{0}$. We set $\omega_{\mathrm{D}}=10 \Delta_{0}$. The current density is given by $\mathbf{j}(\mathbf{r}) \propto \operatorname{Im} \sum_{j}\left[f\left(E_{j}\right) u_{j}^{*}(\mathbf{r}) \nabla u_{j}(\mathbf{r})+\{1-\right.$ $\left.\left.f\left(E_{j}\right)\right\} v_{j}(\mathbf{r}) \nabla v_{j}^{*}(\mathbf{r})\right]$. We consider an isolated vortex under the following conditions. (a) The system is a cylinder with a radius $R$. (b) The Fermi surface is cylindrical, appropriate for the materials such as $\mathrm{NbSe}_{2}$ and high- $T_{c}$ cuprates. (c) The pairing has isotropic $s$-wave symmetry. Thus the system has a cylindrical symmetry. We write the eigenfunctions as $u_{j}(\mathbf{r})=u_{n \mu}(r) \exp \left[i\left(\mu-\frac{1}{2}\right) \theta\right]$ and $v_{j}(\mathbf{r})=v_{n \mu}(r) \exp \left[i\left(\mu+\frac{1}{2}\right) \theta\right]$ with $\Delta(\mathbf{r})=\Delta(r) \exp [-i \theta]$ in polar coordinates, where $n$ is a radial quantum number and the angular momentum $|\mu|=\frac{1}{2}, \frac{3}{2}, \frac{5}{2}, \cdots$. We expand the eigenfunctions in terms of the Bessel functions $J_{m}(r)$ as $u_{n \mu}(r)=\sum_{i} c_{n i} \phi_{i\left|\mu-\frac{1}{2}\right|}(r)$ and $v_{n \mu}(r)=$ $\sum_{i} d_{n i} \phi_{i\left|\mu+\frac{1}{2}\right|}(r)$ with $\phi_{i m}(r)=\frac{\sqrt{2}}{R J_{m+1}\left(\alpha_{i m}\right)} J_{m}\left(\alpha_{i m} r / R\right)$, $\left(i=1,2, \cdots, N\right.$, and $\alpha_{i m}$ is the $i$-th zero of $\left.J_{m}(r)\right)$. The $\mathrm{BdG}$ is reduced to a $2 N \times 2 N$ matrix eigenvalue problem [3]. Our system is characterized by $k_{\mathrm{F}} \xi_{0}$, which is a key parameter of the present problem.

In Fig. 1, the calculated spatial variation of $\Delta(r)$ is displayed for various $T$. It is seen that as $T$ decreases, the core size $\xi_{1}$ defined by $\xi_{1}^{-1}=\lim _{r \rightarrow 0} \Delta(r) / r$ shrinks and the oscillatory spatial variation with a wave length $\sim 1 / k_{\mathrm{F}}$ becomes evident in $\Delta(r)$ [1.:3]. The physical reason for this Friedel-like oscillation lies in the following facts. All eigenfunctions $u_{n \mu}(r)$ and $v_{n \mu}(r)$ contain a rapid oscillation component with $1 / k_{\mathrm{F}}$. At lower $T$ the lowest bound states, whose oscillation amplitude is large near the core, dominate physical quantities. We note that the oscillatory behavior can always appears at sufficiently low $T$ irrespective of values of $k_{\mathrm{F}} \xi_{0}$. We also mention that a similar oscillatory spatial variation around a vortex core in the Bose condensate of ${ }^{4} \mathrm{He}$ is found theoretically, due to the roton excitations 17.

The associated supercurrent $j_{\theta}(r)$ and the field $H(r)$ are shown in Fig. 2. Reflecting the above oscillation, $j_{\theta}(r)$ also exhibits a weak oscillation around $r=0.2-0.5 \xi_{0}$. It is difficult to see the oscillation in $H(r)$, because it is obtained by integrating $j_{\theta}(r)$ via the Maxwell equation $\nabla \times \mathbf{H}=\frac{4 \pi}{c} \mathbf{j}(\mathbf{r})$, resulting in a smeared profile. It is also seen that the position of the maximum of $j_{\theta}(r)$ becomes shorter as $T$ decreases. These features quite differ from those obtained within the Ginzburg-Landau (GL) framework [11, 18].

The $T$ dependence of $\xi_{1}(T)$ for various $k_{\mathrm{F}} \xi_{0}$ values is shown in Fig. 3. Coinciding with Kramer and Pesch (1] for $s$-wave pair and Ichioka et al. [8] for $d$-wave pair, $\xi_{1}(T)$ decreases almost linearly with $T$, that is, $\xi_{1}(T) / \xi_{0} \sim T / T_{c}$ except at extremely low $T$. An important difference from these quasiclassical theories [1],8] appears at lower $T$. At a lower $T<T_{0} \simeq T_{c} /\left(k_{\mathrm{F}} \xi_{0}\right)$, where the quantum limit is realized, the shrinkage of the core size stops to saturate, and the saturated value is estimated as $\xi_{1} / \xi_{0} \sim\left(k_{\mathrm{F}} \xi_{0}\right)^{-1}$.

According to the $\mu \mathrm{SR}$ experimental data [10], the core radius in $\mathrm{NbSe}_{2}$ shows a strong $T$ dependence, while that in YBCO with $T_{c}=60 \mathrm{~K}$ is almost $T$-independent below $\sim 0.6 T_{c}$. This seemingly contradicting result can be understood as follows. The strong $T$ dependence in $\mathrm{NbSe}_{2}$ is the usual KP effect corresponding to the curves for larger $k_{\mathrm{F}} \xi_{0}$ in Fig. 3. At lower $T$ than $T_{0}$ estimated as $\sim 100 \mathrm{mK}\left(k_{\mathrm{F}} \xi_{0} \sim 70\right)$, the shrinkage must saturate (the above experiment is done above $\sim 2 \mathrm{~K}$ ). As for the YBCO data, since the estimated $k_{\mathrm{F}} \xi_{0}$ is small $(\sim 4$ 12 for $\mathrm{YBCO}$ with $T_{c}=90 \mathrm{~K}$ ), the saturation is already attained at a relatively high $T$ such as shown in Fig. 3 . Thus the absence or weakness of the KP effect in YBCO is simply attributable to the fact that the quantum-limit temperature $T_{0}$ is quite high.

Reflecting the shrinkage of the core radius, the boundstate energies $E_{\mu}$ increases as $T$ decreases. This $T$ dependent $E_{\mu}$ shift, due to the KP effect, and its saturation at lower $T$ may lead to a nontrivial $T$ dependence in thermodynamic and transport properties.

In Fig. 4 , we plot the energy levels $E_{\mu}$ of the lowlying bound states $\left(\mu=\frac{1}{2}, \frac{3}{2}, \cdots, \frac{13}{2}\right)$ as a function of $k_{\mathrm{F}} \xi_{0}$, at sufficiently low $T\left(T / T_{c}=0.01\right)$ where increasing of the energy levels saturates. It is seen that in large- $k_{\mathrm{F}} \xi_{0}$ region, the bound states densely pack inside the gap $\Delta_{0}$, allowing us to regard them as continuous ones. This is the case where the quasiclassical approximation [1, 8] validates. In small- $k_{\mathrm{F}} \xi_{0}$ region, where the quantum effect is important even at high $T$, only a few bound states remain within the low-energy region. We find that even in small- $|\mu|$ region, the spacing between the energy levels $E_{\mu}$ is not constant, but rather becomes narrower as $|\mu|$ increases. The often adopted formula $E_{\mu} / \Delta_{0}=2 \mu /\left(k_{\mathrm{F}} \xi_{0}\right)$ or $2 \mu /\left(k_{\mathrm{F}} \xi_{1}\right)$ due to Caroli et al. [2], or $E_{\mu} / \Delta_{0}=\left(2 \mu / k_{\mathrm{F}} \xi_{0}\right) \ln \left[\xi_{0} / 2 \xi_{1}\right]$ by Kramer and Pesch in the limit $\xi_{1} \ll \xi_{0}[$ [] $]$ do not satisfactorily explain our self-consistent results. Instead, our result is empirically fitted to a formula $E_{1 / 2} / \Delta_{0}=\left(0.5 / k_{\mathrm{F}} \xi_{0}\right) \ln \left[k_{\mathrm{F}} \xi_{0} / 0.3\right]$ for 
large $k_{\mathrm{F}} \xi_{0}$ as shown in the dotted curve in Fig. 4.

In Fig. 5, the spectral evolution, i.e., the spatial variation of LDOS, which is calculated by $N(\mathbf{r}, E) \propto$ $\sum_{j}\left[\left|u_{j}(\mathbf{r})\right|^{2} f^{\prime}\left(E-E_{j}\right)+\left|v_{j}(\mathbf{r})\right|^{2} f^{\prime}\left(E+E_{j}\right)\right]$, is shown for $k_{\mathrm{F}} \xi_{0}=8$ at low temperature $T=0.05 T_{c}$. It is well contrasted with that of the higher $T$ case by Gygi and Schlüter [3] (see, for comparison, Fig. 15 in Ref. 33] where $k_{\mathrm{F}} \xi_{0} \sim 70$ and $T \simeq 0.13 T_{c}$, calculated under the two-dimensional Fermi surface). As lowering $T$, because of the quantum effects, the thermally smeared spectral structure drastically changes and becomes far finer one around the vortex. The spectra are discretized inside the gap and consist of several isolated peaks, each of which precisely corresponds to the bound states $E_{\mu}$ $\left(|\mu|=\frac{1}{2}, \frac{3}{2}, \cdots\right)$. Reflecting the oscillatory nature of the eigenfunctions $u_{\mu}(r)$ and $v_{\mu}(r)$ with the period $1 / k_{\mathrm{F}}$, the spectral evolution also exhibits the Friedel-like oscillation as seen from Fig. 5 .

To show clearly the particle-hole asymmetry of the LDOS of Fig. 5, which is another salient feature, we present in Fig. 6 the spectra at the vortex center $r=0$ and $0.2 \xi_{0}$ [We can barely see the asymmetry in Wang and MacDonald [13. (see Fig. 3(a) in Ref. [13)]. At the center $r=0$, the bound-state peak with $E_{1 / 2}$ appears only at $E>0$ side, because the eigenfunction $u_{1 / 2}(r=0) \neq 0$, which consists of the Bessel function $J_{0}(r=0)(\neq 0)$, and all others for $\left|E_{\mu}\right|<\Delta_{0}$ vanish at $r=0$. At $r=0.2 \xi_{0}$, the other bound-state peaks are seen. The particle-hole asymmetry in the vortex bound states appears even if the normal-state density of states is symmetric. These features are subtle [3] or absent [15] in the previous calculations. This asymmetry around the vortex is quite distinctive, should be checked by STM experiments, and may be crucial for the Hall conductivity in the mixed state.

Let us argue some of the available experimental data in the light of the present study. The lowest bound state level $E_{1 / 2} / \Delta_{0}$ is estimated by Maggio-Aprile et al. 12 for YBCO with $T_{c}=90 \mathrm{~K}\left(E_{1 / 2}=5.5 \mathrm{meV}\right.$ and $\left.\Delta_{0}=20 \mathrm{meV}\right)$, yielding $k_{\mathrm{F}} \xi_{0} \sim 4$. Since it implies that $\xi_{0}$ is only of the order of the crystal-lattice constant, we should caution that Maggio-Aprile et al. 12] take their data for the spectral evolution every $10 \AA$ apart near the core, thus the important spatial information on LDOS might be lost. So far the existing STM data 12, 16, 19 taken at the vortex center are almost symmetric about $E=0$, e.g., on $\mathrm{NbSe}_{2}$ at $T=50 \mathrm{mK}$ [16]. The reason why the so-called zero-bias peak is centered just symmetrically at $E=0$ is that $k_{\mathrm{F}} \xi_{0}$ is large and $T$ is too high to observe the quantum effects.

We emphasize that in any clean $s$-wave type-II superconductors at appropriately low $T\left(<T_{0} \simeq T_{c} /\left(k_{\mathrm{F}} \xi_{0}\right)\right)$, one can observe these eminent characteristics associated with the quantum effects. For example, a typical A-15 compound $\mathrm{V}_{3} \mathrm{Si}$ [5] with $T_{c}=17 \mathrm{~K}, \xi_{0} \simeq 60 \AA\left(k_{\mathrm{F}} \xi_{0} \simeq\right.$
12), and a borocarbide $\mathrm{LuNi}_{2} \mathrm{~B}_{2} \mathrm{C}$ [19 with $T_{c}=16 \mathrm{~K}$, $\xi_{0} \simeq 80 \AA$ are the best candidates to check our results.

In summary, we have analyzed the vortex core structure and the related quasiparticle energy spectrum by self-consistently solving the BdG equation for an isolated vortex in a clean $s$-wave type-II superconductor, focusing on the low- $T$ quantum effects. We have found the far richer structure in the pair potential, supercurrent, and LDOS than what one naively imagines from the corresponding calculations done at high $T$ or $k_{\mathrm{F}} \xi_{0} \gg 1$ [3, 15], and pointed out experimental feasibility to observe it.

The widely used working hypothesis for the vortex core of a rigid normal rod with the radius $\xi_{0}$ 11 must be cautiously used for the clean superconductors of interest: the magnetic field distribution probed by neutron diffraction [20] or $\mu \mathrm{SR}$ [10] through the magnetic form factor analysis based on the GL theory must be taken with caution. Detailed investigations of various mysteries associated with the vortices, e.g., the thermal Hall conductance [4] belong to future work.

We would like to thank J. E. Sonier and A. Yaouanc for useful discussions.

FIG. 1. The spatial variation of the pair potential $\Delta(r)$ normalized by $\Delta_{0}$ around the vortex for several temperatures and $k_{\mathrm{F}} \xi_{0}=16$. The length $r$ is measured by $\xi_{0}$.

FIG. 2. The current distribution normalized by $c \phi_{0} /\left(8 \pi^{2} \xi_{0}^{3} \kappa^{2}\right)$ for several temperatures, where $\phi_{0}$ is the flux quantum and $\kappa(\gg 1)$ is the GL parameter. The inset shows the field distribution normalized by $\phi_{0} /\left(2 \pi \xi_{0}^{2} \kappa^{2}\right)$. The temperatures are the same as in Fig. 1 , and $k_{\mathrm{F}} \xi_{0}=16$.

FIG. 3. The $T$ dependence of the vortex radius $\xi_{1}$ normalized by $\xi_{0}$ for several $k_{\mathrm{F}} \xi_{0}(=1.2,2,4$, and 16 from top to bottom).

FIG. 4. The lowest seven bound-state energies $E_{\mu}$, normalized by $\Delta_{0}$, as a function of $k_{\mathrm{F}} \xi_{0}$, at enough low temperature $T / T_{c}=0.01$. The dotted line is a fitting curve (see the text).

FIG. 5. The spectral evolution $N(E, r)$ at $T / T_{c}=0.05$ and $k_{\mathrm{F}} \xi_{0}=8$. It is normalized by the normal-state density of states at the Fermi surface. $E$ and $r$ are measured by $\Delta_{0}$ and $\xi_{0}$, respectively.

FIG. 6. The local density of states $N(E, r)$ at $r=0$ (solid line) and $0.2 \xi_{0}$ (dotted line). $T / T_{c}=0.05$ and $k_{\mathrm{F}} \xi_{0}=8$. 
[1] L. Kramer and W. Pesch, Z. Phys. 269, 59 (1974).

[2] C. Caroli et al., Phys. Lett. 9, 307 (1964); Phys. Kondens. Mater. 3, 380 (1965).

[3] F. Gygi and M. Schlüter, Phys. Rev. B 43, 7609 (1991).

[4] K. Krishana et al., Phys. Rev. Lett. 75, 3529 (1995). See also, K. Krishana et al., Science 277, 83 (1997).

[5] See, e.g., R. Corcoran et al., Phys. Rev. Lett. 72, 701 (1994).

[6] See, e.g., A. van Otterlo et al., Phys. Rev. Lett. 75, 3736 (1995); N. B. Kopnin and G. E. Volovik, condmat/9706082, A. I. Larkin and Yu. N. Ovchinnikov, condmat/9708202 These authors try to understand vortex dynamics in terms of the quasiparticle excitations in a vortex core.

[7] G. E. Volovik, JETP Lett. 58, 455 (1993).

[8] The KP effect for $d$-wave pair: M. Ichioka et al., Phys. Rev. B 53, 15316 (1996).

[9] S. G. Döttinger et al., Phys. Rev. B 55, 6044 (1997).

[10] J. E. Sonier et al., Phys. Rev. Lett. 79, 2875 (1997); 79, 1742 (1997).

[11] A. L. Fetter and P. C. Hohenberg, in Superconductivity, edited by R. D. Parks (Marcel Dekker, New York, 1969), Chap. 14.

[12] I. Maggio-Aprile et al., Phys. Rev. Lett. 75, 2754 (1995).

[13] Y. Wang and A. H. MacDonald, Phys. Rev. B 52, 3876 (1995).

[14] Y. Morita et al., Phys. Rev. Lett. 78, 4841 (1997); Phys. Rev. Lett. 79, 4514 (1997); Europhys. Lett. 40, 207 (1997). See also, M. Franz and M. Ichioka, Phys. Rev. Lett. 79, 4513 (1997).

[15] N. Hayashi et al., Phys. Rev. Lett. 77, 4074 (1996); Phys. Rev. B 56, 9052 (1997). For vortex lattice case, M. Ichioka et al., Phys. Rev. B 55, 6565 (1997).

[16] H. F. Hess et al., Phys. Rev. Lett. 62, 214 (1989); Phys. Rev. Lett. 64, 2711 (1990); Physica B 169, 422 (1991); H. F. Hess, Physica C 185-189, 259 (1991).

[17] S. Giorgini et al., Phys. Rev. Lett. 77, 2754 (1996), and earlier references therein.

[18] H. E. Brandt, Phys. Rev. Lett. 78, 2208 (1997).

[19] Y. De Wilde et al., Phys. Rev. Lett. 78, 4273 (1997).

[20] M. Yethiraj et al., Phys. Rev. Lett. 78, 4849 (1997); M. R. Eskildsen et al., Phys. Rev. Lett. 79, 487 (1997). 


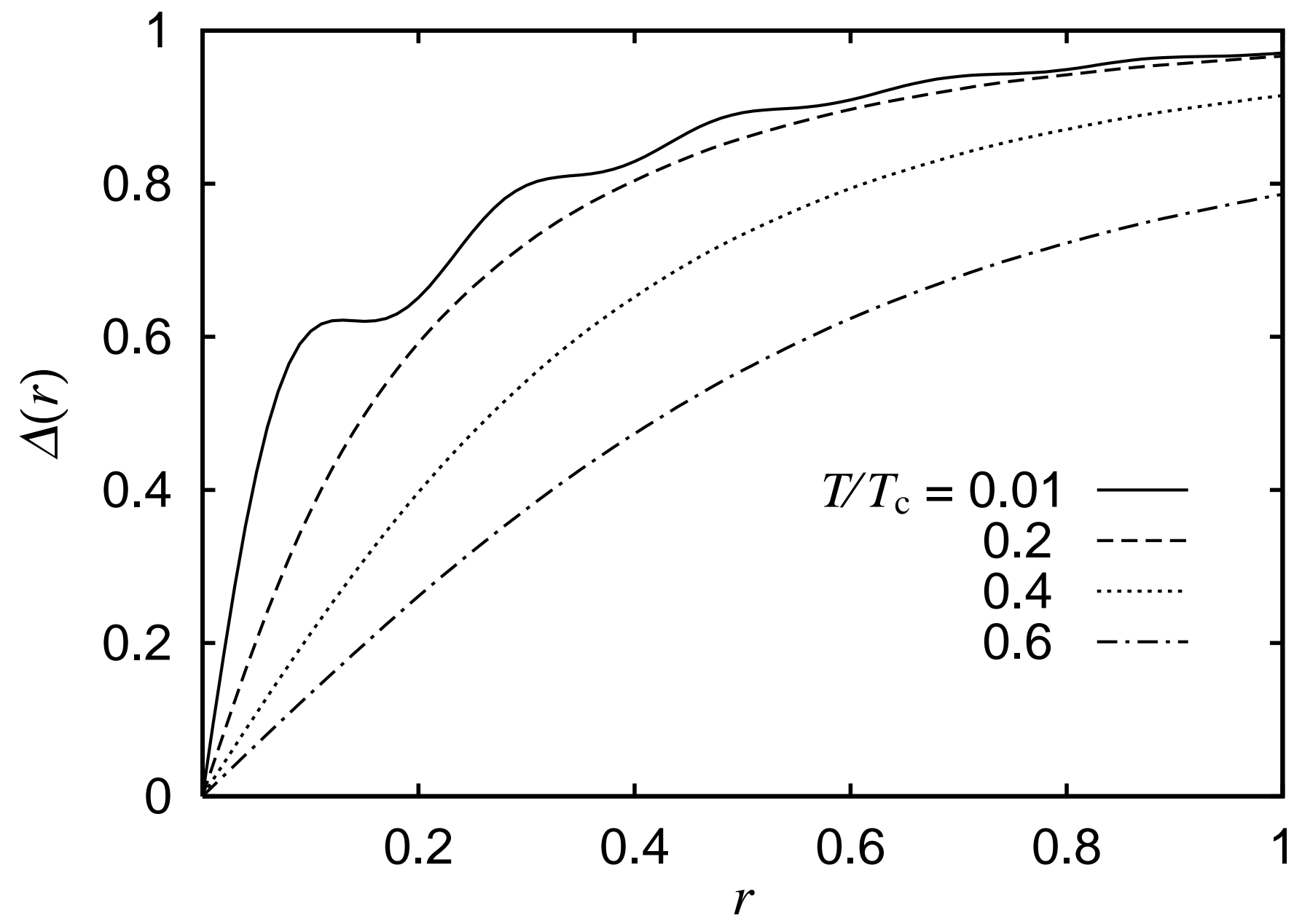

Fig.1 Hayashi et al. 


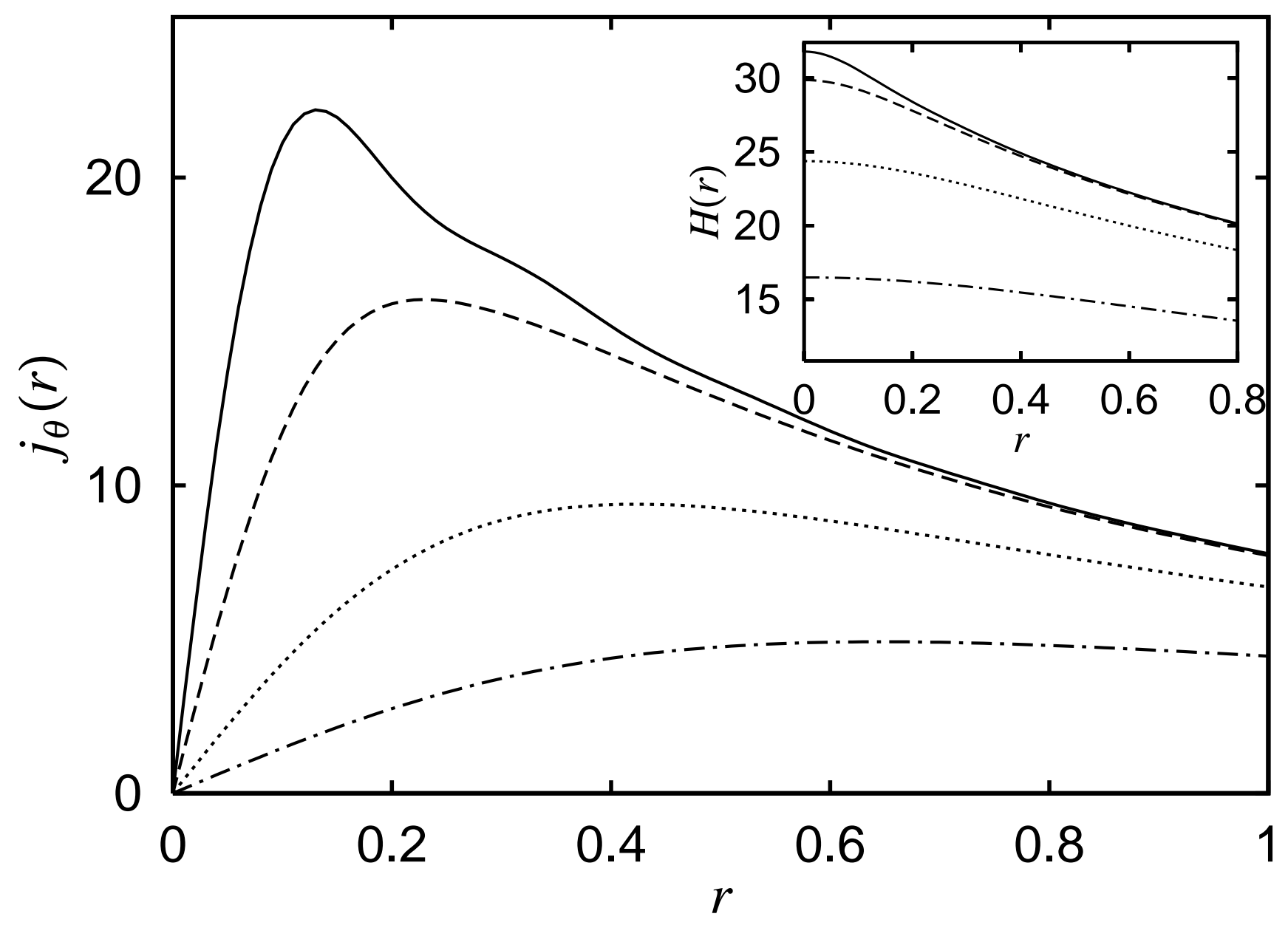

Fig.2 Hayashi et al. 


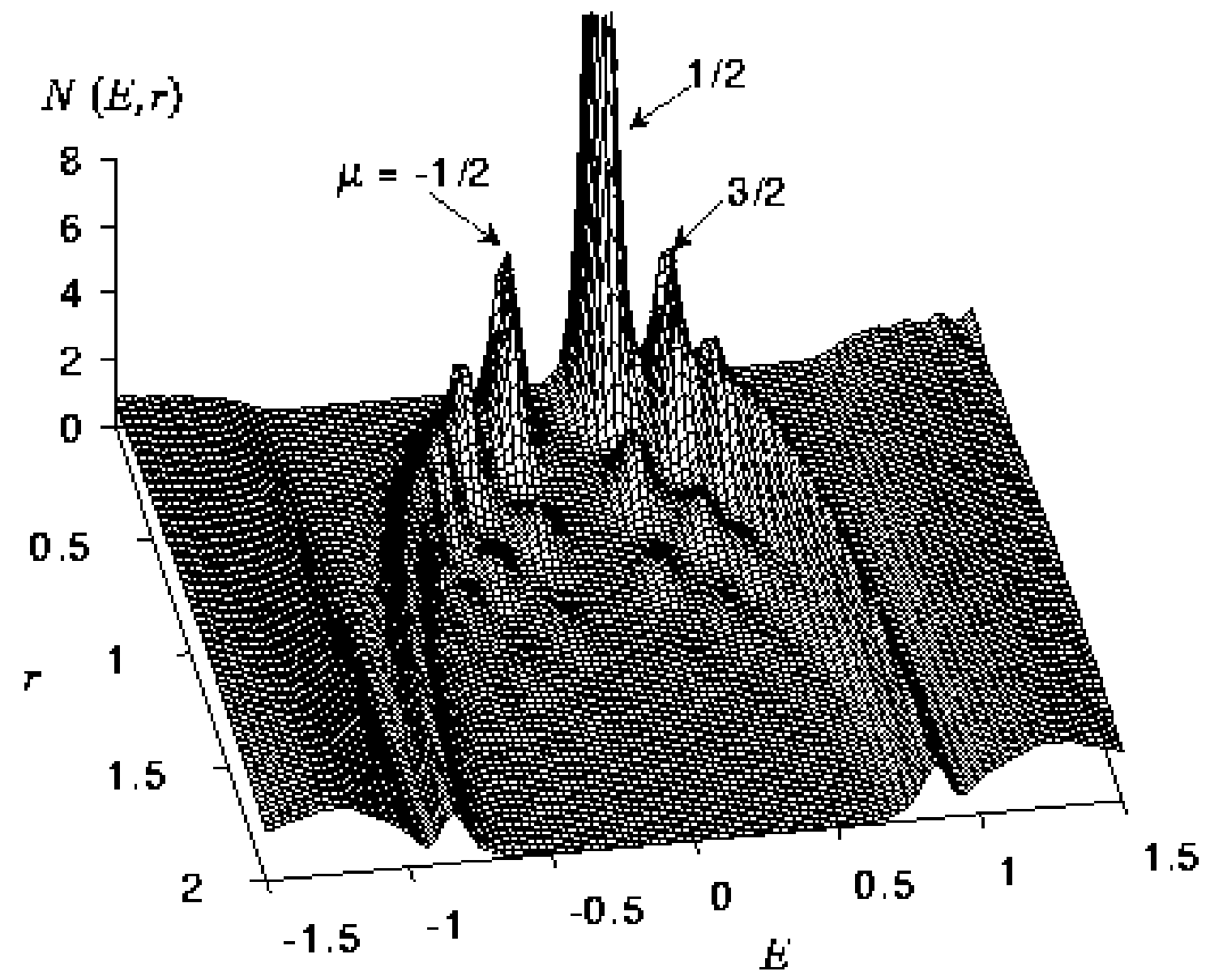

Iijg.5 llayashi etal. 


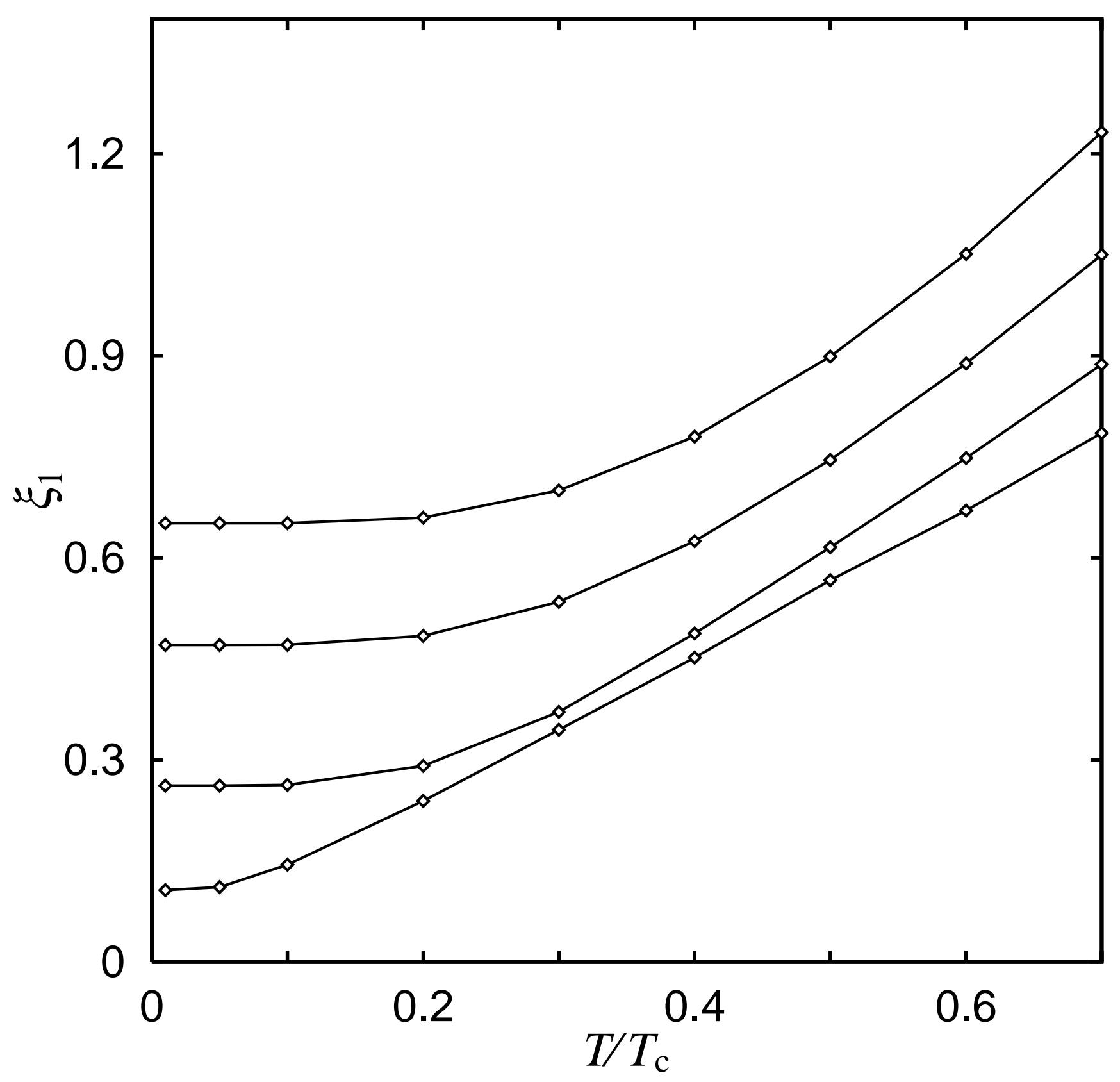

Fig.3 Hayashi et al. 


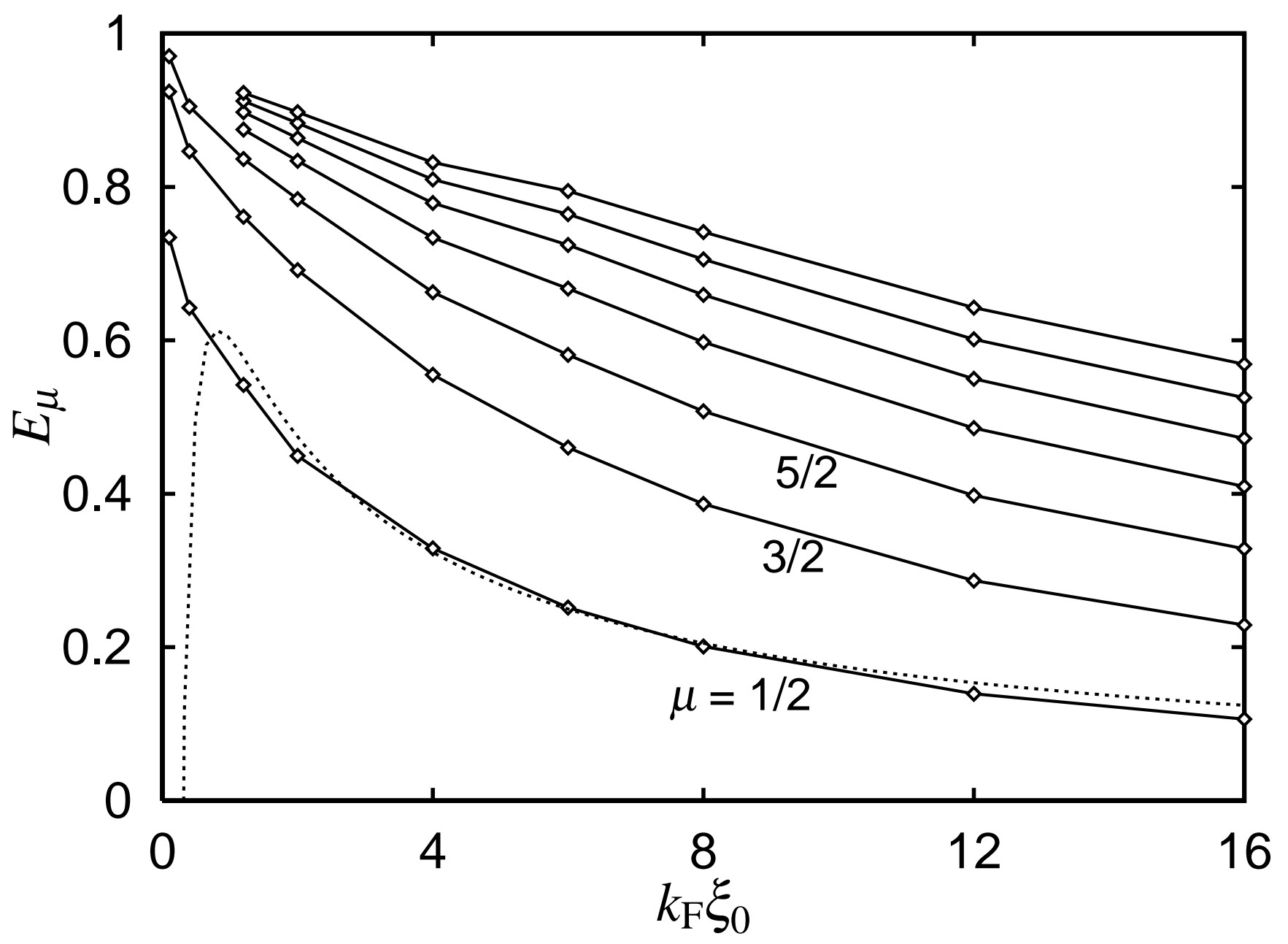

Fig.4 Hayashi et al. 


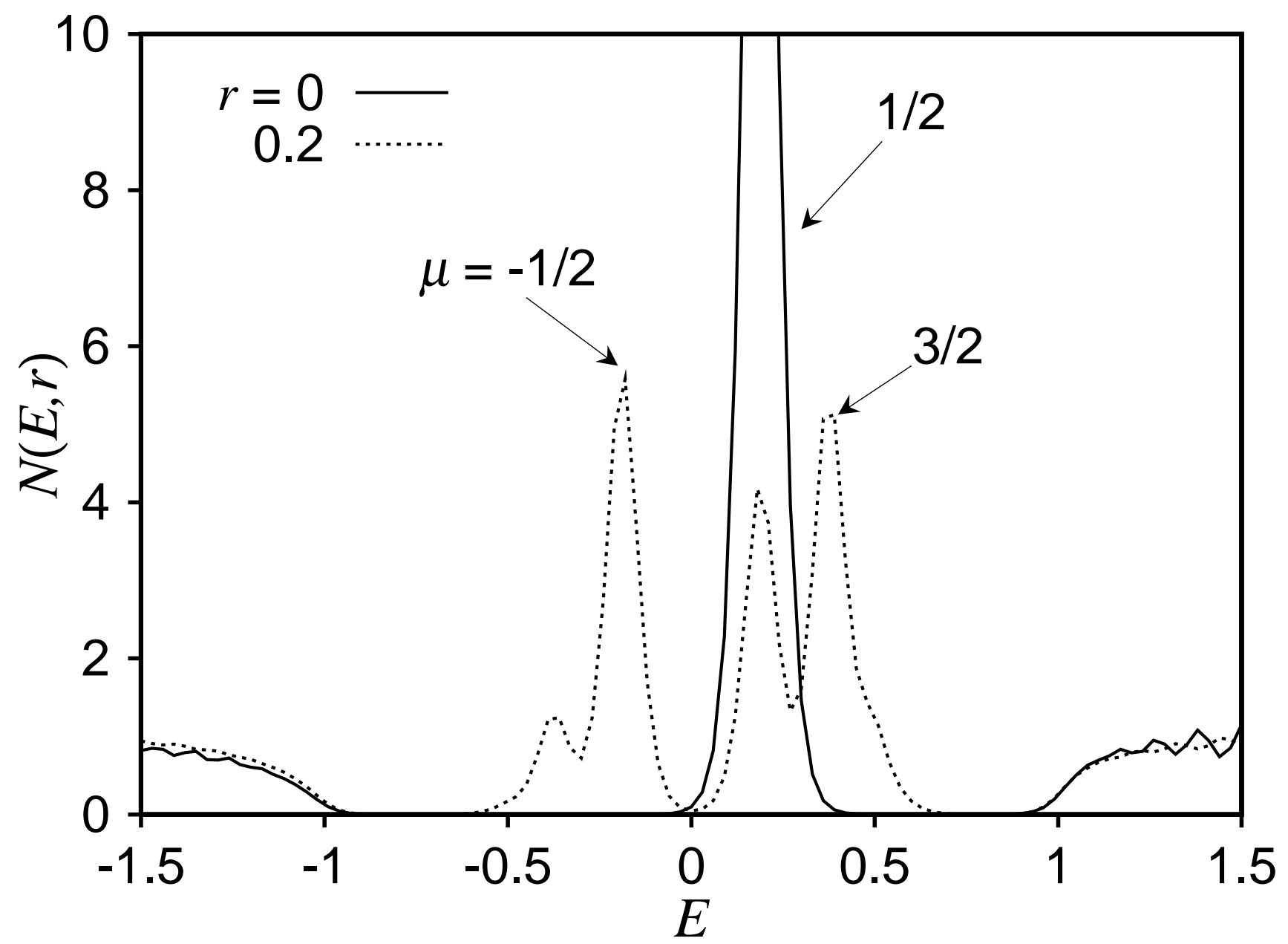

Fig.6 Hayashi et al. 